\title{
On the Physical Impairments Constraint in OBS Networks
}

\author{
Oscar Pedrola, Davide Careglio, Member, IEEE, Mirosław Klinkowski*, Josep Solé-Pareta \\ CCABA, Universitat Politècnica de Catalunya, 08034 Barcelona, Spain \\ * Z-14, National Institute of Telecommunications, 51-501 Wrocław, Poland \\ e-mail: \{opedrola, careglio,pareta\}@ac.upc.edu,mklinkow@itl.waw.pl
}

\begin{abstract}
The introduction of sub-wavelength switching is one of the main objectives of the future transparent optical mesh networks. One strong candidate is Optical Burst Switching (OBS) which improves the resource utilisation by setting up very short lived circuits (bursts). Nonetheless, introducing optical transparency in the optical layer has an important impact on the network performance. In fact, the signal propagates through a transparent optical path accumulates the effect of a variety of quality degrading phenomena which limits the system reach. Translucent optical networks are emerging as a promising solution for bridging the gap between opaque and transparent networks. In this paper we investigate these alternatives in OBS networks and test the effectiveness
\end{abstract} of the translucent solution.

Keywords: Optical Burst Switching, physical layer impairments, routing algorithms, regenerator placement.

\section{INTRODUCTION}

The current network architecture migration aims at decreasing the overall cost (both CAPEX and OPEX) and the operations effort, and at improving the scalability and the suitability to future services. This trend enforces to the development of optical networks which is currently experiencing an evolution from opaque to transparent architecture [1].

In an opaque network, each node is equipped with Optical Electrical Optical (OEO) interfaces in such a way that any optical signals terminate to undergo an OEO conversion and an electronic processing. This approach allows a full independence between the network and the physical layer simplifying the network design and control. On the contrary, it requires a large amount of OEO devices greatly increasing the network cost.

In transparent optical network, the optical signal remains in the optical domain bypassing the intermediate nodes optically. This approach reduces considerably the cost since neither OEO conversions nor electronic processing is required at each node along a path. A further step toward scalable transparent optical mesh network is the introduction of sub-wavelength switching at optical layer using technologies such as Optical Packet Switching (OPS), Optical Burst Switching (OBS) or Optical Data-unit Switching (ODS). Among these potential solutions, OBS appears the best candidate attracting most of the current research works.

Unfortunately, despite of the recent advances in optical technologies that are fostering the deployment of such transparent optical networks, the physical layer impairments (PLIs) of the optical transmission systems prevent it from taking place, at least, in the short-medium term [1]. In fact, while transparency implies that the physical layer must support end-to-end communication, actually the transmission reach of optical signals is limited due to the accumulation of PLIs such as cross-talk, chromatic and polarization-mode dispersion, opticalfiber nonlinearities, amplified spontaneous emission (ASE) noise, etc. Such PLIs cause signals' quality of transmission (QoT),- for example the Bit Error Rate (BER), - to drop beyond an acceptable level making the correct reception at destination unviable. To go beyond this limit, re-timing, re-shaping, and re-amplifying the optical signal by means of $3 \mathrm{R}$ regeneration is essential. Unlikely, optical $3 \mathrm{R}$ regenerators are still not available and transparency is feasible only in limited-size networks.

For that very reason, translucent (or semi-transparent) optical networks are emerging as a promising solution for bridging the gap between opaque and transparent networks. Indeed, translucent networks combine features of both opaque and transparent networks strategically placing OEO regeneration (i.e. by means of OEO conversion) only at selected points in the network [2]. The so-called regenerator placement (RP) problem arises: an RP algorithm must be able to find both the minimum number of regenerators and their exact location for a network topology so that for every source-destination node pair a path can be established. This approach eliminates much of the required electronic processing and allows a signal to remain in the optical domain for much of its path.

In this paper we investigate the current research efforts to include the physical layer impairment constraints in the design and operation processes of the OBS networks and present some exemplary results comparing opaque, transparent and translucent solutions. The rest of the paper is organized as follows. In Section II, we survey the previous work in this topic. In Section III, we provide a simple routing and regenerator placement algorithm for the planning phase and test its effectiveness compared to the opaque and transparent cases. Section IV concludes the paper.

\section{RELATED WORK}

An opaque network is characterized by OEO regenerations at every node. In such a case, the physical layer must only ensure that any transmission link connecting two nodes allows a signal QoT above the acceptable level. 
The main scope of the network layer is to find an available route between a source and a destination disregarding about the PLIs. This approach is usually indicated as the most expensive one [3].

In both transparent and translucent optical networks, the network and the physical layer cannot be decoupled (as in opaque networks) and a cross-layer design is necessary. The traditional problem of routing must account the signal QoT in the path computation process in the so called impairment aware routing (IA-R). Two cases can be faced. During the planning phase, the traffic demand is already known at least partially; therefore the decisions can be taken offline using static IA-R algorithms. The other case, whereby traffic demands are assumed to arrive in a dynamic fashion, is referred to as the online or dynamic IA-R problem.

Besides, in the translucent optical networks, there are the additional problems of regenerator placement (RP) and regenerator allocation (RA). Because of the high cost of the regenerators (remind that they consist of OEO devices), the minimization of the number of such components present in the network is crucial to the problem's success. In the planning phase, RP consists of selecting which nodes of the network have regeneration capabilities and how many signals can be regenerated at those nodes. In contrast, in the operation phase, RA tries to determine how the already placed regenerators are used in a dynamic scenario. It is known that RP is an NPcomplete problem [4] and heuristic methods are generally employed. Recent studies in OCS (e.g. [5]) show that better network performance can be achieved by treating the RP and the routing problem together in the so-called RRP problem.

The majority of research efforts spent in such problems have been mainly devoted to the optical circuit switched (OCS) networks. A comprehensive literature review can be found in [6].

In contract, the vast majority of the works in OBS consider that either an ideal physical layer or optical regenerators at every channel, port and switching node of the network are available (i.e., fully transparent OBS). Recently, however, owing to the increasing interest on assessing the effect of the PLIs in the optical networks field, we find few interesting works that involve the PLI constraint in the evaluation of the OBS network performance. For example, some impairment-aware scheduling policies with the aim of minimizing the burst loss probability are presented in [7]. Another interesting study that incorporates PLIs in the definition of an algorithm for distributing manycasting services over an OBS network can be found in [8]. An extensive study that evaluates the design and maximum size and throughput for OBS core nodes considering the effects of a range of PLIs such as amplifier noise, crosstalk of WDM channels, gain saturation and dynamics can be found in [9]. However, in this work, all nodes are equipped with $\mathrm{O} / \mathrm{E} / \mathrm{O}$ wavelength converters (which also perform electrical regeneration), and thus, an opaque OBS network is being considered. No RRP algorithms have been available in literature.

\section{TRANSLUCENT OBS ARCHITECTURE}

In this work we analyse the advantageous of a translucent OBS architecture compared to the opaque and transparent cases. To this aim, firstly we define a translucent OBS node and a model to compute the signal QoT; secondly, we propose a simple RRP algorithm for the planning phase and test its effectiveness compared to the opaque and transparent cases.

\subsection{Architecture}

The node architecture here used is based on the model proposed in [9], where an opaque Tune-and-Select (TAS) OBS network solution is considered. We modify this opaque TAS OBS core node architecture by replacing each inline electrical wavelength converter with a block consisting of a tuneable laser and a wavelength conversiontype SOA (WC-SOA) device. Additional ports are connected to a limited number R of regenerators in such a way that all wavelengths from all input ports have the same privileges when requesting a regenerator, and thus, fairness in the access to the regenerator pool is provided by this architecture.

The impact of PLIs is captured by considering the optical signal to noise ratio (OSNR) at the receiving end as in [10]. Although there exist many other PLIs, either linear or non-linear, in this model we consider only the amplified spontaneous emission (ASE) noise introduced by both the EDFA and SOA amplifiers as the significant signal impairment factor. The OSNR consists of two main components, namely the link and node OSNR that we denote as $L$ and $N$ respectively. We can compute $P_{o s n r}$ for an optical end-to-end path traversing $k$ links by using the following equation

$$
P_{o s n r}=1 /\left(\sum_{i=1}^{k} \frac{1}{L_{i}}+\sum_{i=1}^{k} \frac{1}{N_{i}}\right),
$$

where $L_{i}$ considers the ASE noise induced by all the (pre- and line) amplifiers spans forming a link while $N_{i}$ takes into account the presence of the SOA switches, SOA converters, splitters, combiners, mux/demux and EDFA booster amplifier.

The $P_{o s n r}$ is then compared to an OSNR threshold $\left(T_{o s n r}\right)$ to determine whether the PLI constraint is fulfilled at the receiver node. 


\subsection{The Regenerator Grouping (RG) algorithm}

In order to test the effectiveness of the translucent OBS network compared to the opaque and transparent architectures, we propose a simple RRP algorithm called Regenerator Grouping (RG). Specifically, given a topology and a traffic matrix, RG provides the explicit paths to be used to route bursts through the network, the placement of regenerators in selected nodes on those paths, and the dimensioning of regenerators in the network.

RG is based on three main phases. The first two phases are the routing and the regenerator placement. For each demand $d$ of the traffic matrix $\mathbf{D}$, they are performed one after the other and iteratively. Although the order of the iteratively processed demands may result in different solutions, still we observed that the algorithm performance does not vary significantly. Thus, we consider an arbitrary order.

For the routing phase, $\mathrm{RG}$ aims at the selection of paths that tends to group the regenerators in nodes as much as possible. First, it checks if there is a path $p_{d}$ for the demand $d$ such that the OSNR requirements are met (i.e. $\left.P_{\text {osnr }}>T_{\text {osn }}\right)$; if yes, it selects the shortest one. Otherwise, the algorithm performs the search for the set of paths $P_{d}$ with the maximal number of regenerators placed so far and, among these paths, it selects the shortest one $p_{d}$.

The regenerator placement phase searches for appropriate location of regenerators in intermediate nodes on path $p_{d}$ is performed. Note that this step is run only if necessary (i.e., whenever $p_{d}$ does not meet the OSNR requirements). First, the algorithm checks if among intermediate nodes $V_{p}$ there are nodes with regenerators already located (for previously processed paths). If so, among those nodes it selects node $v$, the nearest one to the middle of the path (with respect to the number of hops), and considers it as a regenerative node for path $p_{d}$. Now the path $p_{d}$ is decomposed into two transparent segments; the segments that do not meet the OSNR requirement are re-segmented again until no more regenerators are needed.

The results of these phases are the set of valid routing paths $p_{d}$ and the set of nodes $R_{d}$ in which the regeneration of an optical burst, when sent on path $p_{d}$, has to be performed.

Since OBS is based on statistical multiplexing, the bursts entering in a node compete in the access to regeneration resources. For this reason, a last phase to compute the number of regenerators per place is needed. In order to determine the number of regenerators required in node $v$ we define a dimensioning function $f\left(\rho_{v}, B\right)$ where $B$ represents some target burst blocking probability and $\rho_{v}$ the load of the bursts traffic entering in node $v$. Under the assumption that any burst may access any regenerator in a node we make use of the inverse of the Erlang B-loss function as the dimensioning function $f$.

\subsection{Simulation scenario}

In this section, we present the performance results of the translucent OBS network architecture compared to the opaque and transparent cases. While RG algorithm is used for the translucent scenario, opaque and transparent scenarios consider the simple shortest path algorithm.

The metric of interest in this study is the overall burst loss probability (BLP). The evaluation is accomplished through a series of simulations which consider the following simulation scenario: (1) simulations are conducted on the JAVOBS [11] network simulator; (2) the Pan-European Core network is the topology considered [12]; (3) channel bit rate is set to $10 \mathrm{Gbps}$; (4) the load is normalised to the link capacity; (5) bidirectional links equipped with 32 channels each; (6) the traffic is uniformly distributed; (7) the OSNR threshold $T_{\text {osn }}$ is $20 \mathrm{~dB}$ (which is fairly enough taking into account the value proposed in [10]); (8) $B$ is set to $10^{-3}$; (9) the set of candidate paths $P_{d}$ for the $\mathrm{RG}$ algorithm is set to 2 .

\subsection{Results}

Figure 1 shows the BLP for the transparent, opaque and translucent scenario. Three different curves are plotted: BLP due to the contention, BLP due to bursts arrived at destination with an OSNR under $T_{\text {osnn }}$, and the total BLP. In addition, the total number of regenerators placed in each case is shown in the table. In the transparent scenario, the nodes are not equipped with regenerators while each node has one regenerator per wavelength and per fibre in the opaque scenario; in the translucent scenario, the regenerators are placed according to the results of the RRP algorithm.

The result of the transparent case makes it clear that the impact of the PLIs in an OBS network is quite severe; losses are completely dominated by bursts dropped due to OSNR. On the other hand, the opaque solution provides the lowest BLP but requires a vast number of regenerators (1472). It is easy to observe that for the proposed translucent architecture, the BLP are dominated by the contention which means that OSNR losses are maintained under control thanks to the regenerators placed in the network.

Figure 2 plots the BLP performance with respect to the number of regenerators placed in the translucent network. Both the transparent and opaque cases are plot and used as benchmarking indicators; as before, they use 0 and 1472 regenerators respectively. As expected, the BLP of the translucent network decreases as the number of regenerators increases; starting with a BLP equal to the transparent case (0 regenerators), after placing few regenerators (around 30), it reaches the performance of the opaque case. It is worth to mention that the slight better BLP of translucent case when more than 30 regenerators are placed is due to the differences in the node 
architectures; the opaque node has in-line regenerators as in [9] whilst the regenerators in the translucent node operate in the feed-back mode.
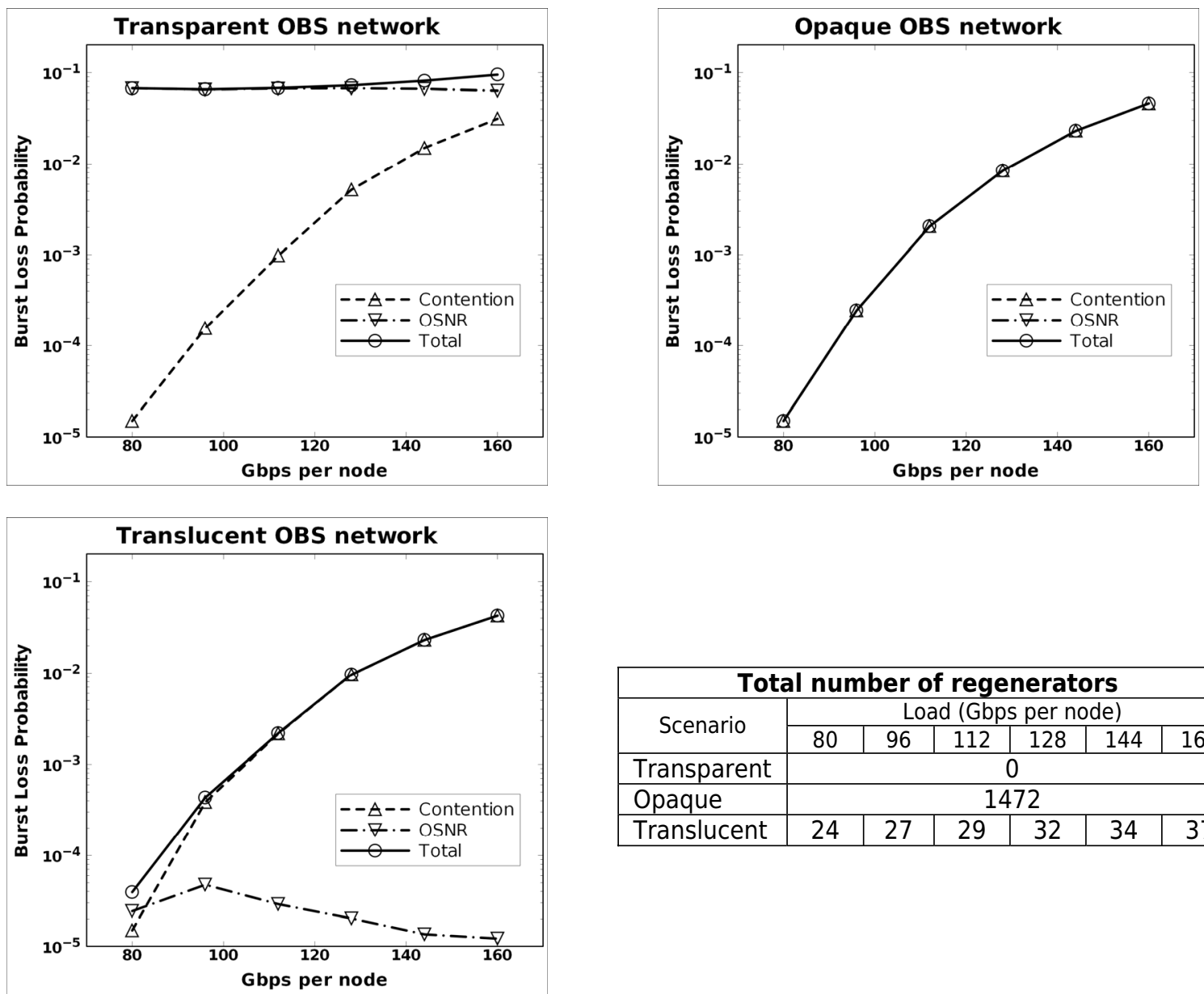

\begin{tabular}{|l|c|c|c|c|c|c|}
\hline \multicolumn{7}{|c|}{ Total number of regenerators } \\
\hline \multirow{2}{*}{ Scenario } & \multicolumn{7}{|c|}{0} \\
\cline { 2 - 7 } & 80 & 96 & 112 & 128 & 144 & 160 \\
\hline Transparent & \multicolumn{7}{|c|}{1472} \\
\hline Opaque & \multicolumn{7}{|c|}{37} \\
\hline Translucent & 24 & 27 & 29 & 32 & 34 & 37 \\
\hline
\end{tabular}

Figure 1. Burst Loss Probability as a function of the load comparing the transparent, opaque and translucent networks.

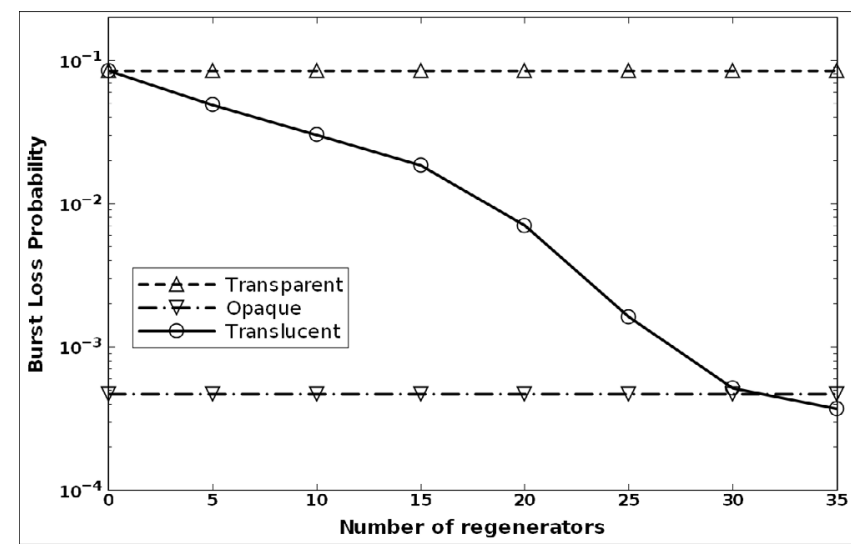

Figure 2. Burst Loss Probability as a function of the total number of regenerators placed in the translucent network; the transparent and the opaque networks are equipped with 0 and 1472 regenerators respectively.

\section{CONCLUSIONS}

In this paper, we have focused on the problem of physical layer impairments in OBS networks. In particular, we have tested the effectiveness of a translucent OBS network architecture where only few nodes are equipped with a limited number of $\mathrm{O} / \mathrm{E} / \mathrm{O}$ regenerators. A simple routing and regenerators placement algorithm called $\mathrm{RG}$ have been proposed and evaluated. Despite the fact that the RG heuristic does not perform an optimal regenerator placement nor an optimal routing decision, it reaches the performance of the opaque OBS network but requiring a reduced amount of regenerators (i.e., 30 vs. 1472). In fact, performance results indicate that the RG heuristic 
successfully maintain negligible bursts losses due to intolerable OSNR with few regenerators and only bursts undergoing to inevitable contentions are lost.

\section{ACKNOWLEDGEMENTS}

The research leading to these results has received funding from the European Community's Seventh Framework Programme FP7/2007-2013 under grant agreement no. 247674 (STRONGEST IP project). The authors of this paper would also like to thank the support from the Spanish Ministry of Science and Innovation under the "COPERNic" project (Ref. TEC2009-13252), the Catalan Government under the contract SGR-1140, and the Polish Ministry of Science and Higher Education under the contract 643/N-COST/2010/0.

\section{REFERENCES}

[1] B. Ramamurthy et al., "Transparent vs. opaque vs. translucent wavelength-routed networks", in Proc. OFC/IOCC, San Diego, CA, USA, Feb. 1999.

[2] G. Shen and R. S. Tucker, "Translucent optical networks: the way forward", IEEE Commun. Mag., vol. 45, no. 2, Feb. 2007.

[3] A. Morea et al., "A critical analysis of the possible cost savings of translucent networks", in Proc. DRCN2005, Island of Ischia, Italy, Oct. 2005.

[4] A. Sen, S. Murthy, S. Bandyopadhyay, "On sparse placement of regenerator nodes in translucent optical network," in Proc. IEEE Globecom 2008, New Orleans, LA, USA, Nov. 2008.

[5] S. Pachnique, T. Paschenda, and P.M. Krummrich, "Physical impairment based regenerator placement and routing in translucent optical networks," in Proc. OFC/NFOEC 2008, San Diego, CA, USA, Feb. 2008.

[6] S. Azodolmolky et al., "A survey on physical layer impairments aware routing and wavelength assignment algorithms in optical networks", Com. Netw., vol. 53, no. 7, May 2009.

[7] Y. Fan, B. Wang, "Physical impairment aware scheduling in optical burst switched networks", Phot. Netw. Commun., vol. 18, no. 2, Oct. 2009.

[8] B.G. Bathula, V.M. Vokkarane, R.R.C. Bikram, "Impairment-aware manycasting over optical burstswitched networks", in Proc. IEEE ICC2008, Beijing, China, May 2008.

[9] H. Buchta and E. Patzak, "Analysis of the physical impairments on maximum size and throughput of SOAbased optical burst switching nodes", IEEE/OSA J. Lightwave Technol., vol. 26, no. 16, Aug. 2008.

[10] H. Pereira et al., "OSNR model to consider physical layer impairments in transparent optical networks", Phot. Netw. Commun., vol. 18, no. 2, Oct. 2009.

[11] O. Pedrola et al., "JAVOBS: A flexible simulator for OBS network architectures", J. Netw., vol. 5, no. 2, Feb. 2010.

[12] S. De Maesschalck et al., "Pan-European optical transport networks: An availability-based comparison", Phot. Netw. Commun., vol. 5, no. 3, May 2003. 